\title{
DISEÑO DE UN MODELO DE EVALUACIÓN DEL DESEMPEÑO DOCENTE BASADO EN LA METODOLOGÍA CAUSAL
}

\author{
DESIGN OF A MODEL TEACHER PERFORMANCE \\ EVALUATION BASED IN THE CAUSAL METHODOLOGY
}

\author{
Hugo Calizaya Calizaya ${ }^{1}$
}

\section{RESUMEN}

Objetivo: La Investigación consiste en diseñar y validar un modelo de evaluación del desempeño docente mediante el modelamiento de ecuaciones estructurales SEM aplicando LISREL.

Método: Se propuso un modelo inicial en base a los resultados de investigaciones y de los datos de los referentes bibliográficos; en los estudios anteriores sólo consideran la influencia de algunas variables sobre la variable resultado. El modelo inicial propuesto consta de catorce constructos: seis exógenos y el resto endógenos con sus respectivas variables observables o medibles y de acuerdo a la metodología causal se traza un diagrama de causa - efecto entre los constructos, cada relación entre variables se codifica para su tratamiento estadístico. Se recogió información de las variables observables mediante cuestionarios previamente validados de tres fuentes: estudiantes, docentes y la autoridad inmediata. La correlación entre las variables observables y los constructos; la correlación entre los constructos se han codificado para construir un sistema de ecuaciones simultaneas; para los cuales debe calcularse sus factores mediante la solución de las mismas. La validación consistió en comparar la matriz de covarianzas esperadas y la matriz de covarianzas reproducidas, se hace una medición de la calidad de los ajustes para lograr los mejores resultados en este caso el modelo.

Resultados: En la investigación se demostró que del modelo propuesto sólo dos constructos: condición del docente y relación autoridad profesor, no tienen relación con los demás; Conclusiones: No deben ser considerados para evaluar el desempeño del docente.

PALABRAS CLAVE: ecuaciones estructurales, evaluación docente, metodología causal

\begin{abstract}
ABSTACT
Objetive: The investigation consisted in investigating and validating an evaluation model of teacher's performance through the modeling of structural SEM equations applying LISREL.

Method: An initial model based on the results of investigations and data from bibliographic referents was proposed. In previous studies they only consider the influence of some variables over the result variable. The proposed initial model consists of fourteen constructs. Six exogenous and six endogenous with their respective observable or measureable variables and in accordance with the causal methodology a diagram of cause is plotted - effect among the constructs, each relationship between the variables is codified for its statistical treatment. Information about the observable variables was collected through previously validated questionnaires from three sources : students, teachers and the immediate authority. The correlation between the observable variables and the constructs, the correlation among the constructs has been codified to build a system of simultaneous equations, for which it is necessary to calculate their factors by solving them. The validation consisted in comparing the matrix of expected covariances and the matrix of reproduced covariances. A measurement of the quality of the adjustments is made to obtain the best results in this case, the model.

Result: In the investigation it was demonstrated that in the proposed model only two constructs, condition of the teacher and relationship authority teacher, were not related to the others.

Conclusion: They must not be considered to evaluate the performance of the teacher
\end{abstract}

KEYWORDS: structural equations, evaluation, teacher, causal methodology

\footnotetext{
1 Mg. en Docencia Universitaria.

Tesis de postgrado para optar el Grado de Dr. en Educación con Mención en Gestión Educativa. Escuela de Postgrado - Universidad Privada de Tacna.

E-Mail: hucali@upt.edu.pe
} 


\section{INTRODUCCIÓN}

Indagar acerca de la calidad de un sistema educativo, supone considerar muchos aspectos, uno de ellos y fundamental es la actividad docente. La evaluación de la docencia universitaria es un problema complejo (Vain 1998), que no puede ni debe, ser abordado desde percepciones reduccionistas que nos impidan visualizar su complejidad.

Para captar en forma adecuada la complejidad de los fenómenos educativos es necesario tomar medidas sobre la cantidad de variables, para ello se han diseñado métodos multivariantes o multivariables que tienen una limitación: solo pueden analizar una relación al mismo tiempo, sin embargo un modelo de ecuaciones estructurales (SEM) permite examinar relaciones para cada subconjunto de variables, aparte de permitir interrelaciones entre algunas variables de estos subconjuntos. Esta técnica nos permite partiendo de un modelo construido en base a una teoría mejorarlo a través de ajustes sucesivos debido a la aplicación del programa estadístico LISREL (Linear Structural Relations), lo que permite reajustar el modelo teórico inicial encontrando uno nuevo y mejor; en este caso un nuevo modelo de evaluación docente (Bisquerra 1989).

Esta investigación responde al propósito esencial, de definir un modelo causal que represente adecuadamente relaciones entre las dimensiones definidas como explicativas del desempeño docente. Este trabajo se realizó con docentes de la carrera profesional de Ingeniería Civil de la Facultad de Ingeniería de la Universidad Privada de Tacna en el año 2011.

El objetivo general de la investigación se centró en formular un modelo de evaluación del desempeño docente basado en un sistema de ecuaciones estructurales.

\section{MATERIALES Y MÉTODOS}

La investigación es: explicativa, cuantitativa, de campo, sincrónica, primaria, pragmática y multivariada por la gran cantidad de variables tratadas. Es un diseño no experimental, relacional ya que no se manipulan las variables, se describen los hechos tal como se presentan y se establecen las relaciones entre variables de estudio.

Para recopilar los datos de la investigación se han diseñado cuestionarios que previamente han sido validados para su aplicación. El campo de aplicación ha sido la Carrera de Ingeniería Civil de la Universidad Privada de Tacna en el último periodo del año 2011, aplicándose a todos los estudiantes; para los docentes, el tamaño de la muestra se determinó de manera no probabilística e intencional; es decir, se tomó como tamaño el número de elementos que cumplieron con criterios establecidos para la presente investigación como ser docentes nombrados y contratados, la muestra fue de 29 profesores.

\section{Factores que intervienen en el desempeño docente}

En base a las investigaciones realizadas por Mateo, Escudero, De Miguel, Mora y Rodríguez (1996) y de Tejedor (1999), y de otros se ha podido plantear un modelo inicial teórico de evaluación docente con los siguientes constructos; Tabla 1:

\section{Tabla 1 Constructos y su simbología para el diagrama} inicial

\begin{tabular}{|l|l|}
\hline CONSTRUCTO & SIMBOLO \\
\hline Estudiantes por asignatura & $\xi_{1}$ \\
\hline Condición del profesor: nombrado o contratado & $\xi_{2}$ \\
\hline Experiencia docente: según años de experiencia & $\xi_{3}$ \\
\hline Naturaleza de la asignatura: teórica, práctica o teórica-práctica & $\xi_{4}$ \\
\hline Ciclo de estudios: I y II; III y IV; V y Vl; VII y VIII; IX y X & $\xi_{5}$ \\
\hline Políticas para el desarrollo docente por las autoridades universitarias & $\xi_{6}$ \\
\hline Cualificación docente & $\eta_{5}$ \\
\hline Relaciones profesor-profesor & $\eta_{6}$ \\
\hline Relaciones profesor-estudiante & $\eta_{7}$ \\
\hline Relaciones autoridad - profesor & $\eta_{8}$ \\
\hline Planificación docente: con 12 indicadores & $\eta_{1}$ \\
\hline Desarrollo de la actividad docente: con 14 indicadores & $\eta_{2}$ \\
\hline Resultados: tasa de rendimiento estudiantil & $\eta_{3}$ \\
\hline Mejora e innovación & $\eta_{4}$ \\
\hline
\end{tabular}

Fuente: Metodología Causal según Enfoque LISREL. Elaboración: Propia.

Jöreskog (1973) para el tratamiento de estas relaciones causales establece, mediante LISREL, una serie de notaciones y convenciones para el diagrama causal y la formulación de las ecuaciones estructurales. Así mismo el procedimiento, condiciones de aplicación de los sistemas de ecuaciones estructurales es tratado ampliamente por Lévy, Varela, Abad (2006). En la Figura 1 se muestra el modelo inicial y su simbología 


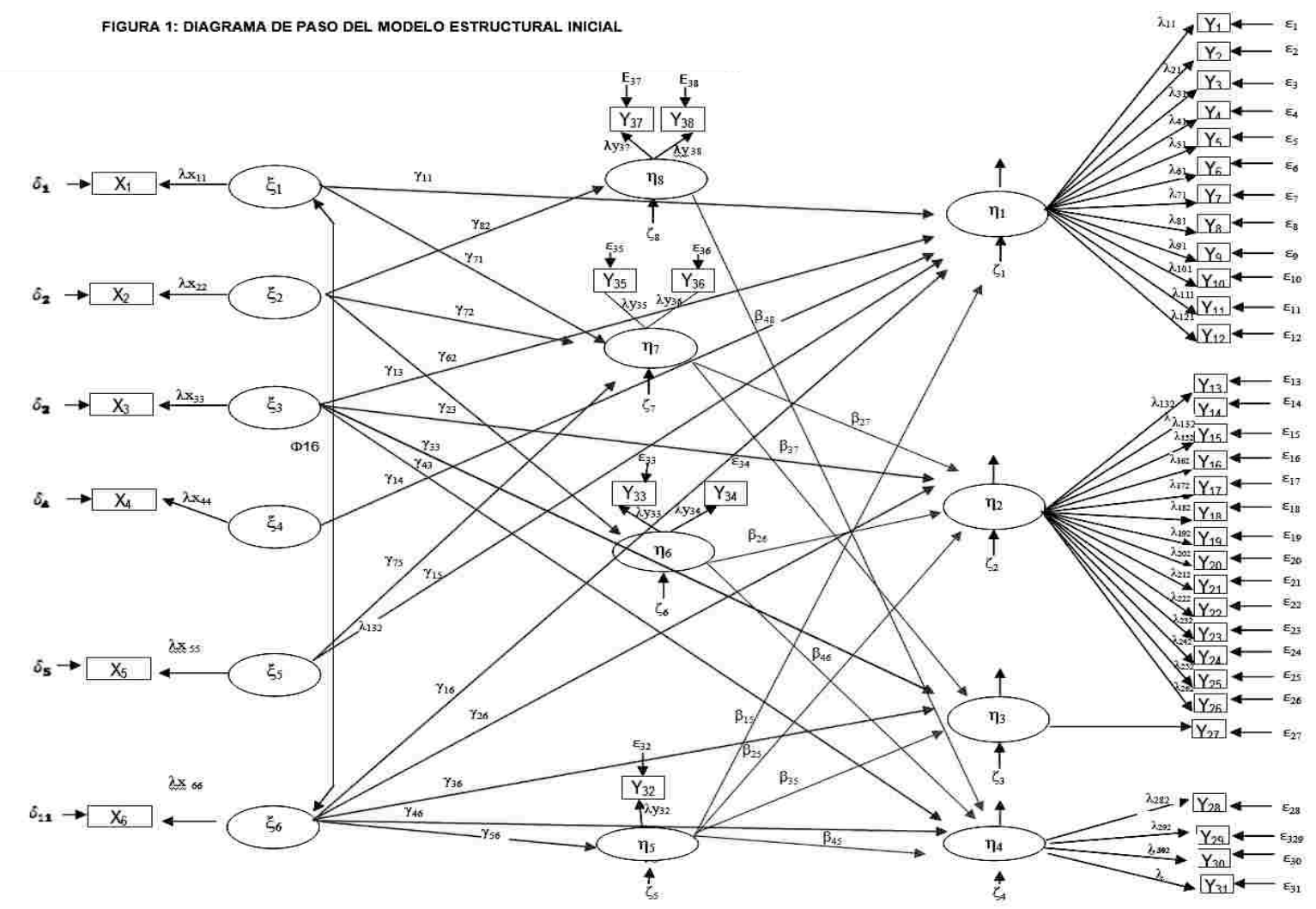

\section{RESULTADOS}

La consistencia interna de los datos fueron evaluados a través del $\alpha$ de Cronbach, resultando el total de la escala 0,989, lo que evidencia una alta consistencia interna y una buena homogeneidad de la escala.

Para asegurar que todos los datos recolectados aportan información se sometió a un análisis descriptivo verificándose que la desviación típica para todos los ítems supera el 0.6 por lo tanto no se puede prescindir de ninguno de ellos. A fin de comprobar que la agrupación teórica realizada a priori es congruente con los criterios empíricos y a fin de reducir la cantidad de variables a introducir en el modelo, se realizó un análisis factorial exploratorio (AFE), para lo cual se incluyeron las treinta y siete variables, con el propósito de desestimar las variables cuya comunalidad resultase no significativa, es decir, variables cuya comunalidad sea inferior al valor 0,6 (valor mínimo requerido según el criterio de significatividad de Kaiser). Se ha eliminado la variable tasa por presentar un valor inferior a 0,6 (Tasa de rendimiento y éxito estudiantil, la cual presentó un valor de 0,543). Mediante la prueba de Kolmogorov Smirnov y la de Shapiro - Wilk, se comprobó que ninguna de las variables sigue una distribución normal.
Concluidos los análisis precursores de las variables que constituían el modelo especificado inicial y habiéndose eliminado las que no ofrecen variabilidad, se presentan las variables que pasaran a formar parte del modelo estructural inicial, agrupadas en sus respectivos constructos; se procedió al calculo de los parámetros del SEM mediante la aplicación de LISREL 8.5. resultando lo siguiente:

\section{Tabla 2. Ecuaciones de medida}

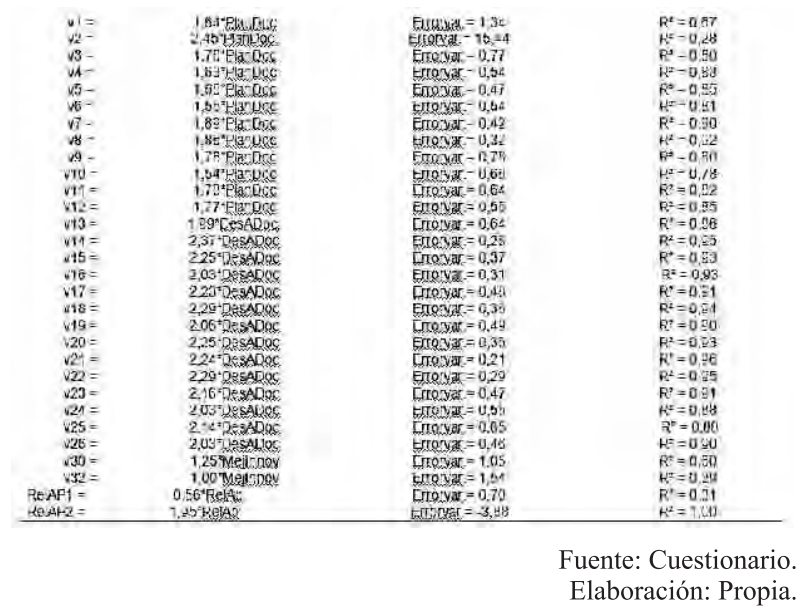


En la Tabla anterior se observa que la variable RelAP2 tiene una varianza negativa $(-3,88)$, por lo cual se eliminó del modelo, procediéndose a una segunda corrida del programa, sin considerar dicha variable (RelAP2), resultando valores positivos para todas las variables.

\section{Índices de Bondad de Ajuste del Modelo}

Los índices obtenidos, son de ajuste absoluto, ya que evalúan directamente el ajuste del modelo: İndice de Bondad de ajuste (GFI = Goodness of Fit Index), el Índice Ajustado de Bondad de Ajuste (AGFI= Adjusted Goodness of Fit Index) y el Índice de la raíz del cuadrado medio del residuo (RMR). El índice GFI puede interpretarse como una medida que determina la proporción de varianza explicada por el modelo, es decir, es comparable al R2 en una regresión lineal. Si además se consideran los grados de libertad y el número de variables observadas del modelo, se obtiene el índice AGFI. El valor que toman estos dos índices se encuentra entre cero y uno, en ambos casos valores cercanos a uno determinan que el modelo tiene muy buen ajuste. Los valores más deseables para el índice RMR están por debajo de 0,05 y entre más cercano a cero es mayor la evidencia de buen ajuste.

En un primer momento de la validación del modelo completo, se le ordena al programa LISREL 8.5 que calcule los parámetros en función de las relaciones teóricas hipotetizadas y explicadas, obteniéndose valores bajos o valores infractores la relación correspondiente a estos ya no son considerados para una segunda corrida.

Al ejecutar LISREL 8.5 por el método de máxima verosilimilitud (ML) y tras 17 iteraciones, los parámetros estimados son los siguientes: se evidencia valores muy bajos relacionados a, CondDoc-CondProf y NatAsigNaturAsi. Así mismo se han producido estimaciones infractoras en los parámetros RelAP1, RelAP2, RelE1, RelE2, RelP1, RelP2 ya que el programa Lisrel no puede calcular sus estimaciones que le corresponden.

Los resultados de la bondad de ajuste del modelo que el valor de ajuste AIC (Model AIC $=8943,06)$ se sitúa próximo al modelo saturado (Saturated AIC $=1980,00)$, el ajuste se considera medianamente aceptable. Así mismo como el valor Expected Cross-Validation Index $($ ECVI $)=$ 11,24 se sitúa más próximo al valor ECVI saturado $(2,49)$ que al valor ECVI independencia $(213,93)$, el ajuste es medianamente aceptable. Los valores NFI, NNFI, PNFI, CFI, IFI, RFI, se encuentra próximamente al valor recomendado, cuyo límite de aceptación es de 0,9.

Estos índices de ajuste nos indican que el modelo precisa una re especificación, por lo que se realizó modificaciones basadas en fundamentos teóricos para lograr un mejor ajuste.

\section{Re especificación del modelo}

La primera modificación fue la eliminación de la relación causal RelAP1 con la variable latente intermedia RelAP por que el programa LISREL arrojó la existencia de una varianza negativa, consecuentemente ello trajo consigo al ejecutar nuevamente el programa un valor ínfimo de relación en la relación RelAP2 con la variable latente intermedia RelAP, por lo que se eliminó la variable latente RelAP con sus enlaces correspondientes.

Al realizar las modificaciones señaladas se observó una baja correlación entre la variable observada exógena CondDoc con la variable latente exógena CondProf, por lo cual también se determinó su eliminación. La no presencia de la variable latente exógena CondProf implicó consecuentemente las eliminaciones de las relaciones causales: RelAp $\rightarrow$ CondProf y RelPP $\rightarrow$ CondProf.

Al hacer estas modificaciones conseguimos mejoras en el modelo. Se hace un nuevo análisis de las estimaciones para los indicadores de las variables latentes exógenas y endógenas mediante el programa LISREL resultando que las ponderaciones de los indicadores en los constructos asignados en la mayoría de los casos resultan idóneas con respecto al Modelo inicial. Asimismo se observa que aparecen los errores de medida de los indicadores de los constructos latentes exógenos estos valores en su mayoría se encuentran dentro de los límites aceptables a excepción del indicador PoliDes.

\section{Evaluación del ajuste del modelo estructural}

En resumen, observamos que los índices de bondad de ajuste del modelo reespecificado se encuentran dentro de los límites aceptables los valores de GFI y AGFI, si bien no llegan a 0,9 , sus valores tienden a aproximarse $(0,69$ y $0,63)$, por lo que se aceptan estas medidas con mucha precaución. En la tabla 3 siguiente se aprecian el valor de los índices de ajuste obtenidos que están dentro de los valores recomendados.

Tabla 3 Otros índices de ajuste del modelo re especificado

\begin{tabular}{|c|c|c|}
\hline Indice & Valor réccomendado. & Vator obtenido \\
\hline $\begin{array}{l}\text { incice de bondad de ajuste } \\
\text { comparado CFI }\end{array}$ & Iguai o mayor que 0,90 & 0,96 \\
\hline $\begin{array}{l}\text { Indice de bondad de ajuste } \\
\text { incremental IFI }\end{array}$ & Iguad a meyor que 0,90 & 0,96 \\
\hline indice de & Entre 0,5 y 0,7 & 0,58 \\
\hline
\end{tabular}

Fuente: Resultados Docentes LISREL 8.5 Elaboración: Propia. 
Las diferencias más notables entre el modelo estructural propuesto y el modelo reespecificado han consistido en la eliminación de algunos paths (conexiones causales) como: RelAP $1 \rightarrow$ RelAP, RelAP $2 \rightarrow$ RelAP, RelE2 $\rightarrow$ RelPE, RelP $1 \rightarrow$ RelPP, V29 $\rightarrow$ MejInnov,
V $30 \rightarrow$ MejInnov, Cond D o c $\rightarrow$ CondProf, $\mathrm{RelAP} \rightarrow$ CondProf $\mathrm{y}$ RelPP $\rightarrow$ CondProf que trajo como resultado la mejora en la parsimonia del modelo, tal como se aprecia en la Figura 2.

Figura 2: Modelo reespecificado con sus parámetros y ajuste

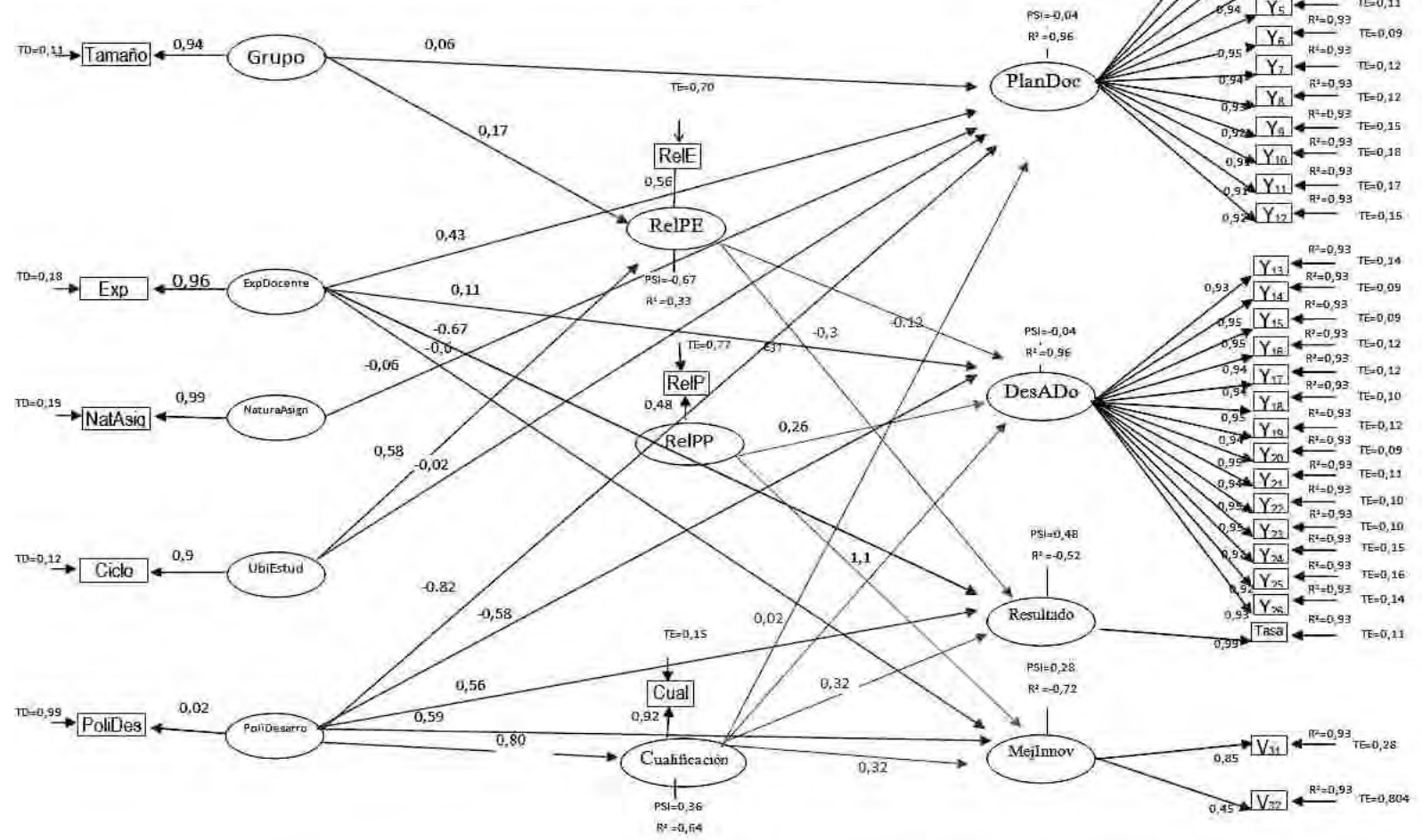

\section{DISCUSIÓN}

Los resultados del presente estudio, basado en un modelo causal, nos permitieron valorar comparativamente varios modelos hipotéticos alternativos que establecían relaciones de causalidad diversa entre las variables latentes o dimensiones de medida estudiadas, las cuales recogen los diversos componentes utilizados por otros investigadores. Resulta interesante observar en el modelo reespecificado (Figura 2) la plausibilidad del efecto directo de las variables grupo, experiencia docente, ciclo y políticas de desarrollo en el constructo endógeno desempeño docente, evidenciado a través de planificación docente, desarrollo de la actividad docente, resultados y mejora e innovación y un efecto mediacional de la variable latente cualificación docente, descartándose varias conexiones causales, como por ejemplo, la relación entre la variable acreditación de nuevas competencias para la comunicación oral o escritas de las lenguas de aplicación para la docencia y la organización o asistencia a cursos de metodología con el constructo endógeno mejora e innovación, en un contexto particular como es la Escuela Profesional de Ingeniería Civil de la UPT, afirmación que no podría inferirse a las demás escuelas, lo que nos permite valorar la variable experiencia en la docencia y considerar variables no consideradas usualmente relevantes al momento de valorar los resultados evaluativos del profesorado, como ser el tamaño del grupo que tiene a su cargo, el ciclo al cual pertenece el grupo e inclusive las políticas de desarrollo de las habilidades y destrezas instauradas por la propia institución, como variables predictoras del desempeño.

\section{REFERENCIAS}

1. BISQUERRA, R. (1989). Introducción conceptual al análisis multivariable. Vol. II, PPU, Barcelona.

2. LEVY, J., VARELA J. (2006) Modelización Con Estructuras De Covarianzas En Ciencias: Temas esenciales, avanzados y aportaciones especiales, Edit. Netbiblo, Madrid. 
3. Mateo, J., Escudero, T., De miguel, M., Mora, J. G., \& Rodríguez, S. (1996) La Evaluación del profesorado. Un tema a debate, Revista de Investigación Educativa.

4. Tejedor, F. y García, A. (1999) Evaluación institucional en la Universidad, En: Revista Gallega de Pisopedagoxia.

5. Vain Pablo D. (1998) La Evaluación de la docencia Universitaria: Un problema complejo. Universidad Nacional de Misiones. 